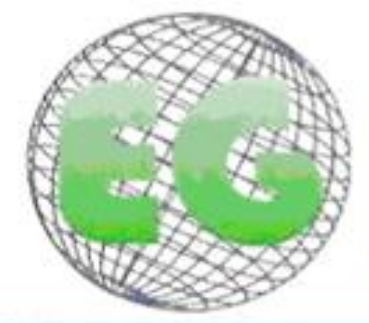

ISSN 1695-6141 $N^{\circ} 37$

www.um.es/egloball

\title{
CLÍNICA
}

\section{Capacidad funcional $y$ nivel cognitivo de adultos mayores residentes en una comunidad en el sur de Brasil}

Capacidade funcional e nível cognitivo de idosos residentes em uma comunidade do sul do Brasil

Functional capacity and cognitive level of elderly residents in a community in southern Brazil

\section{*Leite, Marinês Tambara ${ }^{* *}$ Castioni, Daniani ${ }^{* * *}$ Kirchner, Rosane Maria ****Hildebrandt, Leila Mariza}

*Enfermera, Doctora en Gerontología Biomédica, docente de la Universidad Federal de Santa Maria Rio Grande do Sul. E-mail: tambaraleite@yahoo.com.br ***Enfermera, egresa del Curso de Enfermería de la Universidad Federal de Santa Maria/Campus Palmeira das Missões. "***Doctora en Ingeniería Eléctrica. Docente de la Universidad Federal de Santa Maria/Campus Palmeira das Missões. ****Enfermera, Máster en Enfermería Psiquiátrica por la EERP/USP. Docente de la Universidad Federal de Santa Maria/Campus Palmeira das Missões Brasil.

\author{
Palabras Clave: Adulto Mayor; Envejecimiento; Salud del Adulto Mayor. \\ Palavras-chave: Idoso; Envelhecimento; Saúde do Idoso. \\ Keywords: Elderly; Aging; Health of the Elderly.
}

\section{RESUMEN}

Objetivo: Evaluar la capacidad funcional y la función cognitiva de los ancianos residentes en el área urbana de una ciudad de Rio Grande do Sul/Brasil.

Método: Estudio cuantitativo, descriptivo y transversal, en el cual participaron 368 adultos mayores. Para la colecta de los datos se utilizó: cuestionario de identificación, Mini-Mental, Índice de Katz y Escala de Lawton. La estadística descriptiva y test exacto de Fisher fueron utilizados en el análisis.

Resultados: Hubo predominio de mujeres $(64,9 \%)$, de $60-70$ años $(43,8 \%)$, casados $(46,5 \%)$, con algún grado de escolaridad $(90,8 \%)$. En cuanto a la capacidad funcional, $85 \%$ son independientes para las actividades instrumentales de la vida diaria (AIVD) y 93,48\% lo son para las actividades de la vida diaria (AVD).

Conclusión: El estudio revela un significativo porcentual de adultos mayores independientes para la realización de las AVD, sin embargo necesitan de ayuda para las AIVD. 


\section{RESUMO}

Objetivo: Avaliar a capacidade funcional e nível cognitivo de idosos residentes na área urbana de um município do Rio Grande do Sul/Brasil.

Método: Estudo quantitativo, descritivo e transversal, do qual participaram 368 idosos. Para a coleta dos dados utilizou-se: questionário de identificação, Mini-Mental, Índice de Katz e Escala de Lawton. A estatística descritiva e teste exato de Fisher foram utilizados na análise.

Resultados: Houve predomínio de mulheres (64,9\%), de $60-70$ anos $(43,8 \%)$, casados (46,5\%), com algum grau de escolaridade (90,8\%). Quanto à capacidade funcional, $85 \%$ são independentes para as atividades instrumentais da vida diária (AIVD) e 93,48\% o são para as atividades de vida diária (AVD).

Conclusão: $O$ estudo revela significativo percentual de idosos independentes para a realização das AVD, porém necessitam de ajuda para as AIVD.

\section{ABSTRACT}

Objective: To evaluate the functional capacity and cognitive level of elderly people living in the urban area of a city in Rio Grande do Sul/Brazil.

Method: Quantitative, descriptive and transversal study; which one had the participation of 368 elderly people. To collect the data were used: identification questionnaire, Mini-Mental, Katz Index and the Lawton Scale. In the analysis were used the descriptive statistic and the Fisher's exact test.

Results: There were a predominance of women (64.9\%), 60-70 years old (43.8\%), married (46.5\%), with some degree of schooling (90.8\%). Regarding functional capacity, $85 \%$ are independent for Instrumental Activities of Daily Living (IADL) and $93.48 \%$ are independent for Activities of Daily Living $(A D L)$.

Conclusion: The study reveals a significant percentage of independent elderly people for ADL performance, but in need of help for IADL.

\section{INTRODUCCIÓN}

Con el aumento del número de amcianos, resultante de la disminución de las tasas de fecundidad y de natalidad, Brasil viene modificando su estructura de edad. Las estimaciones de envejecimiento humano son de que, en 2050, habrá cerca de 50 millones de personas con edad igual o superior a 60 años, lo que supone el $19 \%$ de la población brasileña ${ }^{(1)}$. Datos del Departamento de Informática del Sistema Único de Salud muestran que en Brasil, en 2012, la población anciana fue de aproximadamente 21 millones. en Rio Grande do Sul llegó a 1,5 millones y en el municipio de Palmeira das Missões/RS alcanzó el número de 4.381 ancianos $^{(1)}$.

El proceso de envejecimiento se acompaña de disminución de las funciones generales del organismo. En lo referente a los cambios del sistema motor, estos pueden ser de origen fisiológico o patológico, en que dichas modificaciones se aceleran después de los 60 años, llevando a la atrofia, pérdida de la fuerza muscular, la acumulación de grasa que puede comprometer la movilidad del individuo(2). Además, algunos trastornos como enfermedades degenerativas centrales, osteoporosis, hiperglucemia y las secuelas del accidente cerebrovascular causan impacto negativo en el rendimiento motor y en deambulación de los ancianos ${ }^{(2)}$. 
Aun así, en el sistema osteoarticular se produce reducción en la producción de las células óseas, lo que se asocia con el desarrollo de la osteoporosis ${ }^{(3)}$. El anciano con osteoporosis es más proclive a tener fracturas, en caso de caídas, y de desarrollar el síndrome post-caída, que incluye la dependencia, la pérdida de autonomía, confusión, inmovilización y depresión y puede llevar a restricciones con miras a la realización de actividades de la vida diaria ${ }^{(3)}$.

Es importante destacar que las enfermedades de carácter crónico a menudo se asocian, tienden a ser expresivas en la vejez y pueden generar discapacidad, afectar a la funcionalidad y a la calidad de vida ${ }^{(4)}$. La capacidad funcional constituye un componente del estudio sobre el estado de salud de los ancianos. Para evaluar el estado funcional es necesario determinar el nivel de independencia para llevar a cabo las actividades de la vida diaria (ADL) y actividades instrumentales de la vida diaria (AIVD). Los resultados pueden mostrar a mayores independientes, parcialmente independientes o dependientes para realizar tales actividades ${ }^{(5)}$. La funcionalidad, también puede estar en relación con el funcionamiento de la persona en su capacidad de autocuidado, la integridad física, el estado intelectual, mental y espiritual e interacciones sociales ${ }^{(6)}$.

El envejecimiento trae consigo también la disminución cognitiva y fisiológica causando aislamiento y la pérdida de autonomía e independencia, porque el rendimiento físico y social de las personas mayores depende de la integridad de todas sus funciones. La pérdida de memoria a corto plazo y la incapacidad para realizar cálculo son indicadores sensibles de la reducción de estas funciones ${ }^{(4)}$.

La demencia es un síndrome crónico y progresivo, resultante de la enfermedad o de la disfunción del cerebro, que provoca cambios en las funciones cognitivas, incluyendo: la memoria, atención y el aprendizaje, pensamiento, orientación, comprensión, cálculo, lenguaje y juicio. Tales modificaciones generalmente vienen acompañadas de alteración del control emocional, comportamiento social o motivación y reducción en la función intelectual ${ }^{(4)}$.

Entre los instrumentos utilizados para evaluar la capacidad cognitiva está el MiniMental State Examination (MMSE), que es la prueba más ampliamente utilizada por ser rápida y fácil de aplicar. Es una de las pocas pruebas validadas y adaptadas a la población brasileña. Resultados muy bajos de la función cognitiva sugieren que deben ser enviados a evaluación neuropsicológica, pues pueden presentar grave patología ${ }^{(4)}$.

Este estudio se realizó con ancianos residentes en zona urbana del municipio de Rio Grande do Sul, Brasil, con el fin de evaluar la capacidad funcional de los mismos. Así, se formula como pregunta de investigación: ¿Cómo es la capacidad funcional, el nivel cognitivo y sus implicaciones en la vida cotidiana de los residentes ancianos en el área urbana del municipio de Rio Grande do Sul/Brasil? Y como objetivo: evaluar la capacidad funcional, el nivel cognitivo y sus implicaciones en la vida cotidiana de los ancianos residentes en el área urbana del municipio de Rio Grande do Sul/Brasil.

\section{MÉTODO}

Este estudio se originó de un subproyecto de la investigación nominada: "La capacidad funcional, causas y consecuencias de las caídas en los ancianos", ligada al 
curso de enfermería del Departamento de Ciencias de la Salud de la Universidad Federal de Santa María/Campus Palmeira das Missões/Rio Grande do Sul/Brasil.

Estudio de carácter cuantitativo, transversal y descriptivo, realizado junto a ancianos de ambos sexos, viviendo en una zona urbana del municipio de Río Grande do Sul/Brasil. Participaron en este estudio 368 ancianos que cumplieron los siguientes criterios de inclusión: de edad de 60 años o más, residente en la zona urbana de la ciudad y ser capaz de responder a la entrevista cognitiva.

Los datos se recolectaron desde marzo de 2011 a julio de 2012, a través de consulta en casa, con la ayuda de cuatro instrumentos, como se describe a continuación: el primero con temas relativos a los datos de identificación, socio-demográficos y de salud. El segundo instrumento fue el Índice de Katz, que investigó el desempeño de las actividades de la vida diaria (AVD), tenía seis preguntas relativas a la posibilidad de bañarse, vestirse, realizar higiene personal, transferencias, continencia y alimentación. La puntuación total fue la suma de preguntas "Sí", contabilizando 1 punto por cada pregunta. El resultado de 6 y 5 puntos representó la independencia para la $A V D, 4$ y 3 puntos fueron dependencia parcial y 2 y 1 puntos la máxima dependencia $^{(7)}$.

El tercer instrumento fue la Escala de Lawton, utilizada para evaluar la capacidad de realizar actividades instrumentales del vivir diario (AIVD), contenida nueve preguntas relacionadas con la capacidad para preparar la comida, realizar las tareas del hogar, servicio de lavandería, manejo de medicamentos, uso del teléfono, manejo de dinero, comprar y utilizar medios de transporte. Cada una de estas acciones ha sido clasificada de 1 a 3 puntos, donde 1 representa la dependencia para tal función, 2 significaba que los ancianos necesitan ayuda y 3 independencia expresada para la función. La puntuación máxima es de 27 puntos y el marcador tiene significado solo individualmente, como carácter comparativo de la evolución del marco general. Para ambos, los elementos se analizaron individualmente entre los miembros de la investigación $^{(7)}$.

El cuarto instrumento utilizado fue el Mini Mental State Examination (MMSE), para evaluar la capacidad cognitiva, siendo evaluados los dominios: orientación temporal, espacial, de la memoria inmediata y de evocación, cálculo, lenguaje-nominación, repetición, comprensión escrita y dibujo. No es adecuado para el diagnóstico, pero sí para indicar las funciones que deben investigarse más. Totaliza 30 puntos, con un mínimo de cero, indica mayor deterioro cognitivo y 30 puntos corresponde a mejor capacidad cognitiva ${ }^{(4)}$. En la evaluación, posee puntuación de corte diferenciado, en que el punto de corte para las personas mayores sin escolaridad es 19, puntuaciones por debajo de este valor son indicadores de déficit cognitivo. Para las personas mayores con escolaridad, el punto de corte es 24 , las puntuaciones por debajo de este valor son indicativas de la declinación cognitiva ${ }^{(8-9)}$.

Los datos se analizaron mediante el paquete estadístico SPSS (Statistical Package for Social Sciences) para Windows, versión 13.0. Para análisis se utilizaron la estadística descriptiva (tablas cruzadas, con frecuencias y porcentajes) y la Prueba Exacta de Fisher. El nivel de significancia de $p<0.05$ fue adoptado para todos los análisis.

El proyecto de investigación fue aprobado por el Comité de Ética de la Universidad Federal de Santa María/RS/BR, bajo el número 0052.0.243.000-11. 


\section{RESULTADOS}

Los participantes del estudio fueron 368 ancianos, $129(35,1 \%)$ eran hombres y 239 $(64,9 \%)$ eran mujeres. Agrupados por grupo de edad la mayoría se clasificó como ancianos de edad joven, había edades comprendidas entre 60 y 70 años 161 (43,8\%), seguida de los que estaban entre 70 y 80 años de edad, 147 (39,9\%) y 80 años o más $60(16,3 \%)$. En cuanto al color de la piel, $277(75,3 \%)$ eran blancos, $77(20,9 \%)$ mixta y $14(3,8 \%)$ negra.

En relación con el estado civil, $171(46,4 \%)$ eran casados, $129(35,1 \%)$ eran viudos y $43(11,7 \%)$ divorciados, $22(6)$ solteros y $3(0,8 \%)$ eran viudos con compañero(a). Con respecto a los niños $343(93,2 \%)$ tenía descendencia y $25(6,8 \%)$ no. En cuanto a intercambio de residencia, $190(51,6 \%)$ residió con compañero(a), $76(20,7 \%)$ vivía sola, $75(20,4 \%)$ vivía con los niños, $9(2,4 \%)$ vivían con nieto y $18(4,9 \%)$ con otras personas. La religión predominante era la Católica $275(74,7 \%)$, seguido por 75 Evangélica $(20,4 \%)$ y otros $18(4,9 \%)$.

Basándonos en los datos obtenidos fue posible identificar que $325(88,3 \%)$ de los encuestados eran retirados y $43(11,7 \%)$ no tenían jubilación. Se comprobó también que $147(39,9 \%)$ de ellos no estaban realizando actividades laborales. De los que permanecían en actividad, $78(21,2 \%)$ trabajaban como empleada doméstica, 74 $(20,1 \%)$ desarrollaban las tareas de casa, 22 (6) en el comercio, $14(3,8 \%)$ en la agricultura, $14(3,8 \%)$ en las actividades artesanales y $19(5,2 \%)$ hizo otro tipo de trabajo.

En lo referente a la educación, $34(9,2 \%)$ de las personas mayores no asistió a la escuela, $273(74,3 \%)$ asistió a escuela primaria incompleta, $16(4,3 \%)$ completa la escuela primaria, $11(3 \%)$ tienen educación secundaria incompleta, $25(6,8 \%)$ tiene la secundaria completa y $9(2,4 \%)$ han completado la educación superior. Con respecto a la presencia de enfermedades $308(83,7 \%)$ reportado haber tenido diagnóstico médico de una patología, con predominio de la enfermedad cardíaca $247(67,1 \%)$ seguido por los trastornos osteoarticulares $74(20,1 \%)$ y enfermedades endocrinas 52 $(14,1 \%)$.

La evaluación para la realización de la AIVD señaló que la mayor dependencia de los ancianos se encontraba en la realización de pequeños trabajos domésticos manuales $(8,2 \%)$. Y el mayor nivel de independencia estaba en tomarla medicación a dosis y tiempo correcto $(92,9 \%)$. Destaca que, en este rango, los items tienen valor individual, de ahí la variación de los resultados que se muestra en la tabla l.

Tabla 1: Clasificación de la capacidad funcional de los ancianos, según la escala de Lawton (AIVD).

\begin{tabular}{|c|c|c|c|}
\hline ARTÍCULO & $\begin{array}{l}\text { Sin ayuda o } \\
\text { con total } \\
\text { independencia } \\
\text { autonomía (\%) }\end{array}$ & $\begin{array}{l}\text { Con autonomía/ } \\
\text { independencia } \\
\text { parcial (\%) }\end{array}$ & $\begin{array}{l}\text { Con } \\
\text { autonomía/ } \\
\text { dependencia } \\
\text { total (\%) }\end{array}$ \\
\hline Utiliza el teléfono & $301(81,8)$ & $39(10,6)$ & $28(7,6)$ \\
\hline El uso de un transporte & $312(84,8)$ & $34(9,2)$ & $22(6,0)$ \\
\hline Realiza las compras & $315(85,6)$ & $32(8,7)$ & $21(5,7)$ \\
\hline
\end{tabular}


Prepara su propia comida

$337(91,6)$

$23(6,3)$

$8(2,2)$

Organiza su casa

$291(79,1)$

$58(15,8)$

$19(5,2)$

Realiza trabajos domésticos y manuales

$298(81)$

$40(10,9)$

$30(8,2)$

Lava y plancha su ropa

Toma el medicamento en dosis y tiempo correctos

$311(84,5)$

$31(8,4)$

Cuida de sus finanzas

Fuente: la investigación de campo.

A través de este estudio, se puede revelar que en lo referente a la AIVD, aproximadamente más del $85 \%$, de los ancianos era independiente, seguido por aquellos con dependencia parcial, alrededor del $9 \%$ y una porción pequeña, aproximadamente $6 \%$ mostró total dependencia.

Con respecto a los resultados obtenidos para la realización de actividades de la vida diaria, Escala de Katz, indicaron que $344(93,48 \%)$ personas mayores eran independientes para todas las funciones, $10(2,72 \%)$ eran dependientes en una función y un encuestado era dependiente para todas las funciones, como se muestra en la tabla II.

Tabla II: clasificación de la capacidad funcional de los ancianos, según la Escala de Katz (AVD).

\begin{tabular}{|c|c|c|}
\hline CLASIFICACIÓN & $\mathrm{N}$ & $\%$ \\
\hline Independiente para todas las funciones & 344 & 93,48 \\
\hline Independiente en todas menos una de las funciones & 10 & 2,72 \\
\hline Dependiente para bañarse y en más de una función & 02 & 0,54 \\
\hline Dependiente para bañarse, vestirse y en más de una función & 01 & 0,27 \\
\hline $\begin{array}{l}\text { Dependiente para bañarse, vestirse, ir al baño y en más de una } \\
\text { función }\end{array}$ & 03 & 0,81 \\
\hline $\begin{array}{l}\text { Dependiente para bañarse, vestirse, ir al baño, moverse y en más de } \\
\text { una función }\end{array}$ & 01 & 0,27 \\
\hline Dependiente para todas las funciones & 01 & 0,27 \\
\hline $\begin{array}{l}\text { Dependiente en al menos dos de las funciones, pero no está en la } \\
\text { clasificación de C, E o F }\end{array}$ & 06 & 1,64 \\
\hline TOTAL & 368 & 100 \\
\hline
\end{tabular}

Fuente: la investigación de campo.

Se identificó en la tabla III, que entre las personas mayores que eran independientes para hacer la AVD, hubo porcentaje significativo que necesitaban ayuda para llevar a cabo la AIVD. Igualmente ocurrió con esas personas mayores que fueron parcial o totalmente dependientes en el logro de la AVD. 
Tabla III: Clasificación de la capacidad funcional para llevar a cabo los AIVDs y los AVDs

\begin{tabular}{|c|c|c|c|c|c|}
\hline \multirow{2}{*}{\multicolumn{2}{|c|}{$\begin{array}{l}\text { Actividades instrumentales de la vida } \\
\text { diaria - Escala de Lawton - AIVDS }\end{array}$}} & \multirow{2}{*}{\multicolumn{4}{|c|}{$\begin{array}{c}\text { Actividades de la vida diaria - Escala de } \\
\text { Katz - AVD } \\
\text { Gradode dependencia }\end{array}$}} \\
\hline & & & & & \\
\hline & & & $\begin{array}{l}\text { funcions } \\
N(\%)^{* *}\end{array}$ & $\begin{array}{l}\text { tunçons } \\
\mathrm{N}(\%)^{\star \star \star}\end{array}$ & $\begin{array}{c}\text { todas } \\
\mathrm{N}(\%)^{* * \star *}\end{array}$ \\
\hline \multirow{3}{*}{$\begin{array}{l}\text { Puede usar el } \\
\text { teléfono }\end{array}$} & No puede & $21(5,7)$ & $4(1,1)$ & $3(0,8)$ & $0(0)$ \\
\hline & $\begin{array}{l}\text { Con ayuda } \\
\text { parcial }\end{array}$ & $31(8,4)$ & $6(1,6)$ & $1(0,3)$ & $1(0,3)$ \\
\hline & Sin ayuda & $292(79,3)$ & $8(2,2)$ & $1(0,3)$ & $0(0)$ \\
\hline \multirow{3}{*}{$\begin{array}{l}\text { Puede moverse } \\
\text { utilizando } \\
\text { algún transporte }\end{array}$} & No puede & $12(3,3)$ & $7(1,9)$ & $3(0,8)$ & $0(0)$ \\
\hline & $\begin{array}{l}\text { Con ayuda } \\
\text { parcial }\end{array}$ & $30(8,2)$ & $2(0,5)$ & $1(0,3)$ & $1(0,3)$ \\
\hline & Sin ayuda & $302(82,1)$ & $9(2,4)$ & $1(0,3)$ & $0(0)$ \\
\hline \multirow{3}{*}{$\begin{array}{l}\text { Puede salir a } \\
\text { hacer compras }\end{array}$} & No puede & $9(2,4)$ & $7(1,9)$ & $4(1,1)$ & $1(0,3)$ \\
\hline & $\begin{array}{l}\text { Con ayuda } \\
\text { parcial }\end{array}$ & $29(7,9)$ & $2(0,5)$ & $1(0,3)$ & $0(0)$ \\
\hline & Sin ayuda & $306(83,2)$ & $9(2,4)$ & $0(0)$ & $0(0)$ \\
\hline \multirow{3}{*}{$\begin{array}{l}\text { Puede preparar su } \\
\text { comida }\end{array}$} & No puede & $1(0,3)$ & $3(0,8)$ & $3(0,8)$ & $1(0,3)$ \\
\hline & $\begin{array}{l}\text { Con ayuda } \\
\text { parcial }\end{array}$ & & 2( & & $0(0)$ \\
\hline & Sin ayuda & 324 (88) & $13(3,5$ & $0(0)$ & $0(0)$ \\
\hline \multirow{3}{*}{$\begin{array}{l}\text { Puede arreglar su } \\
\text { casa }\end{array}$} & $\begin{array}{l}\text { No } p \\
\text { Con }\end{array}$ & $11(3)$ & $3(0,8)$ & $4(1,1)$ & $1(0,3)$ \\
\hline & parcial & $49(13,3)$ & $8(2,2)$ & $1(0,3)$ & $0(0)$ \\
\hline & Sin ayuda & $248(77,2)$ & $7(1,9)$ & $0(0)$ & $0(0)$ \\
\hline \multirow{3}{*}{$\begin{array}{l}\text { Puede hacer } \\
\text { trabajos manuales } \\
\text { y domésticos }\end{array}$} & $\begin{array}{l}\text { No puede } \\
\text { Con avuda }\end{array}$ & $20(5,4)$ & $4(1,1)$ & $5(1,4)$ & $1(0,3)$ \\
\hline & & $34(9,2)$ & $6(1,6)$ & $0(0)$ & $0(0)$ \\
\hline & Sin ayuda & $290(78,8)$ & $8(2,2)$ & $0(0)$ & $0(0)$ \\
\hline \multirow{3}{*}{$\begin{array}{l}\text { Puede lavar y } \\
\text { planchar su ropa }\end{array}$} & No puede & $15(4,1)$ & $4(1,1)$ & $5(1,4)$ & $1(0,3)$ \\
\hline & $\begin{array}{l}\text { Con ayuda } \\
\text { parcial }\end{array}$ & & & & $0(0)$ \\
\hline & Sin ayuda & $303(82,6)$ & $8(2,2)$ & $0(0)$ & $0(0)$ \\
\hline \multirow{3}{*}{$\begin{array}{l}\text { Puede tomar su } \\
\text { medicación en } \\
\text { dosis y momento } \\
\text { adecuado }\end{array}$} & No puede & $1(0,3)$ & $1(0,3)$ & $2(0,5)$ & $1(0,3)$ \\
\hline & parcial & $13(3,6)$ & $4(1,1)$ & $2(0,5)$ & $0(0)$ \\
\hline & Sin ayuda & $328(89,6)$ & $13(3,6$ & $1(0,3)$ & $0(0)$ \\
\hline
\end{tabular}




$\begin{array}{llcccc}\text { Puede cuidar de sus } & \text { No puede } & 3(08) & 4(1,1) & 2(0,5) & 1(0,3) \\ \text { Finanzas } & \text { Con ayuda } & & & & \\ & \text { Parcial } & 27(7,3) & 5(1,4) & 3(0,8) & 0(0) \\ & \text { Sin ayuda } & 314(85,3) & 9(2,4) & 0(0) & 0(0)\end{array}$

* Independencia de todas las actividades; ${ }^{*}$ Dependiente para un máximo de dos funciones; *** Dependiente 3 y 5 funciones dependientes **** Dependiente para todas las funciones; Fuente: la investigación de campo.

Los resultados relativos a la AVD se agruparon en dos categorías: los ancianos que tienen independencia para llevar a cabo diariamente actividades y ancianos quienes tenían dependencia total o parcial. Los datos del MMSE se agruparon en ancianos con deterioro cognitivo y sin deterioro cognitivo, según nivel educativo.

Tabla IV: Distribución de ancianos, según datos del MEEM y capacidad funcional (AVD).

\begin{tabular}{|l|l|l|c|}
\hline & Independencia & & \\
\hline & para todos & Dependencia & \\
\hline Nivel cognitivo & actividades & parcial o total & Total \\
\hline Con deterioro & $121(33,2)$ & $17(4,7)$ & $138(37,8)$ \\
\hline Sin deterioro & $220(60,3)$ & $7(1,9)$ & $227(62,2)$ \\
\hline Total & $341(93,4)$ & $24(6,6)$ & $365(100,0)$ \\
\hline
\end{tabular}

$\mathrm{P}<0,001$ (prueba exacta de Fisher) ${ }^{*}$ Tres datos en blanco

Fuente: Estudio de campo.

Desde el cruce de los datos de la capacidad funcional de las variables y condiciones cognitivas, se puede observar que de 341 (93,4\%) de ancianos independientes para llevar a cabo la AVD, 220 (60.3 \%) no mostraron deterioro cognitivo. De los $24(6,6 \%)$ ancianos que tienen dependencia parcial o total, 17 (4,7\%) tenían deterioro cognitivo.

\section{DISCUSIÓN}

Mediante el análisis de los datos socio-demográficos de este estudio se observó que la mayor parte eran mujeres, en edades de 60-70 años, se casaron y tuvieron algún grado de escolaridad. El predominio de las mujeres puede estar asociado con una mayor longevidad de éstas, en comparación con los hombres, y en el contexto de la feminización del envejecimiento, un fenómeno resultante de la menor exposición de las mujeres a ciertos factores de riesgo que los hombres ${ }^{(10)}$.

Otro aspecto estudiado es la escolaridad, verificándose un alto número de ancianos con ninguno o limitado número de años de estudio formal. Esta es una condición social desfavorable para ellos, ya que puede influir en el acceso a los servicios de salud, participación social y oportunidades en la comprensión de su tratamiento y cuidados personales, entre otros ${ }^{(11-12)}$.

En lo referente a la residencia, hubo predominio de acianos que residía con un cónyuge, pareja, hijos o nietos. Esto denota que la familia también se configura como una organización importante para el apoyo emocional y social a los ancianos, especialmente cuando necesitan cuidados ${ }^{(12)}$. 
Con respecto a la capacidad funcional de los ancianos, la mayoría $(93,8 \%)$ mostró independencia, tanto para la realización de la AIVD como para las AVD. Estos datos difieren de los resultados de la encuesta para estimar la prevalencia de la capacidad funcional y factores asociados en población de edad avanzada en el sur de Brasil, que encontró que el $37,1 \%$ de los ancianos tenía limitaciones en la capacidad funcional ${ }^{(13)}$. La actividad física regular contribuye al mantenimiento de la aptitud física en individuos mayores, además de mitigar y revertir la pérdida de masa muscular ${ }^{(13)}$. Cabe destacar que los mayores participantes en este estudio, en su mayor parte, participaron activamente en las actividades del grupo, éste podría ser uno de los factores que contribuyeron a mantener su capacidad funcional.

También, en cuanto a las AVD, un estudio desarrollado con 310 ancianos, identificó que el $91 \%$ presentó una patología y en lo referente a la AVD, independencia 86,8\% y $13,2 \%$ había presentado dependencia parcial o total. Ya, a través del índice que evalúa el AIVD, el resultado demostró que $47,4 \%$ de los ancianos tenían independencia y $52,6 \%$ se encontraban con otros niveles de dependencia ${ }^{(14)}$.

En Goiânia-Goiás/Brasil, investigación realizada con 388 personas mayores, demostró que 70,9\% tiene al menos una enfermedad, el 65,2 \% era independiente, $31,7 \%$ parcialmente dependiente y $3,1 \%$ tenía dependencia total. En cuanto a la AIVD, 39,4\% eran considerados en términos generales, independientes totales, $45,7 \%$ dependientes parciales y $14,9 \%$ total dependientes ${ }^{(15)}$. Los datos de estos estudios muestran un mayor porcentaje de personas mayores con dependencia total o parcial, tanto en AVD o la AIVD, divergiendo de la presente investigación, que señala que la mayoría de las personas mayores es independiente.

Uno de los elementos evaluados por la Escala de Lawton se relaciona con la capacidad de conseguir tomar sus medicamentos en el tiempo y dosis correcta, 92,9\% de los ancianos encuestados declaró que sí. Esta situación es importante de ser observada, porque una gran parte de los ancianos hace uso de los medicamentos y se asocia con numerosos eventos adversos. La enfermera debe investigar este aspecto en el curso de la consulta de enfermería de tal manera que se puede facilitar el tratamiento y prevención de iatrogénicas ${ }^{(16)}$.

En cuanto al nivel cognitivo, la investigación ha evaluado a los ancianos a través de la medida de independencia funcional (MIF) y el MEEM. De los mayores encuestados $46,3 \%$ mostró demencia severa, seguida de 31,3\% que tenían demencia leve y $22,4 \%$ con demencia moderada. El promedio de MIF para las personas mayores sin déficit cognoscitivo fue 107,7 puntos y, para aquellos con déficit de 63,2 puntos. Esto demuestra que el déficit cognitivo afecta el desempeño de la $\operatorname{AVD}^{(17)}$.

En este estudio también se puede comprobar que una peor capacidad cognitiva puede asociarse con una limitada capacidad funcional. Ante estos resultados los profesionales de la salud, en particular el personal de enfermería, deberían proponer intervenciones dirigidas a la autonomía e independencia de la persona de edad avanzada, promover el desarrollo de sus habilidades de forma independiente, posibilitando el envejecimiento activo, en la que los ancianos pueden cuidar de sí mismos $^{(18)}$.

Capacidad funcional y cognitiva baja puede desencadenar, en la persona de edad avanzada, empeoramiento de la calidad de vida. Cuando se trata de las personas mayores que ya tienen presencia de deterioro cognitivo y funcional se recomiendan 
acciones con el fin de prevenir el desarrollo de más pérdidas. Por lo tanto, es aconsejable adoptar hábitos de vida saludable, la práctica de ejercicio físico, dieta equilibrada, mantenimiento de la convivencia social y estimular las actividades intelectuales con lecturas y juegos.

Para el mantenimiento de la capacidad funcional y cognitivo de las personas mayores, la enermería puede desarrollar estrategias que busquen incentivarlos en el reconocimiento de nuevos proyectos y reclamaciones futuras según su potencial ${ }^{(16)}$. De este modo, el personal de enfermería, especialmente enfermeras, tienen papel importante porque puede intervenir con eficacia en el cuidado de los ancianos, con miras a la preservación de su estado de salud con la máxima autonomía posible y la independencia, junto con su familia y su entorno social.

\section{CONCLUSIÓN}

Los resultados de este estudio mostraro que hubo un predominio de las mujeres $(64,9 \%)$ de $60-70$ años $(43,8 \%)$, casadas (46,5\%), con algún grado de escolaridad $(90,8 \%)$. En cuanto a la capacidad funcional, $85 \%$ son independientes para las actividades instrumentales de la vida diaria (AIVD) y 93,48\% para las actividades de la vida diaria (AVD). Se observó que el déficit cognitivo contribuye a la disminución de la capacidad funcional de los ancianos.

El estudio revela un porcentaje significativo de ancianos independientes para llevar a cabo la AVD, sin embargo, en necesidad de ayuda para la AIVD. La mayoría tienen capacidad funcional, es decir, es independiente, y una pequeña porción tiene dependencia total.

Se considera que este estudio tenía sus limitaciones, ya que participaron personas mayores que viven en área urbana de un único municipio, que no puede retratar la realidad de una región o estado. Aliada a esto, es pertinente para desarrollar estudios con un mayor número de personas mayores y de distintos contextos regionales y municipales.

\section{REFERÊNCIAS}

1. Brasil. Ministério da Saúde. DATASUS - Informações de saúde-demográficas e sociodemográficas 2013 [acesso em 2013 Mai 05]. Disponível em: http://www2.datasus.gov.br/DATASUS/index.php?area=02.

2. Diogo MJD, Neri AL, Cachioni M. Saúde e qualidade de vida na velhice. Campinas, SP, Ed., Alínea, 2009, 236 p.

3. Organização Mundial da Saúde (OMS). Relatório global da OMS sobre prevenção de quedas na velhice. Secretaria de Estado da Saúde, São Paulo, 2010. [acesso em 2013 Mai 04]. Tradução do documento original: Letícia Maria de Campos. Disponível em: http://www.who.int/ageing/projects/falls prevention older age/en/ index.html

4. Brasil. Ministério da Saúde. Envelhecimento e saúde da pessoa idosa. Secretaria de Atenção à Saúde, Departamento de Atenção Básica. - Brasília: Ministério da Saúde, 2007 [acesso em 2013 Mai 04]. Disponível em: http://bvsms.saude.gov.br/ bvs/publicacoes/abcad19.pdf

5. Eliopoulos C. Enfermagem gerontológica. $7^{\text {a }}$ ed., Porto Alegre, Artmed, 2011, 568p. 
6. Brasil. As causas sociais das iniquidades em saúde no Brasil. Relatório final da comissão nacional sobre determinantes sociais da saúde. Abril de 2008. [acesso em 2013 Mai 06]. Disponível em: http://bvsms.saude.gov.br/bvs/publicacoes/causas sociais iniquidades.pdf

7. Freitas EV, Py L, Neri AL, Cançado FAXC, Gorzoni ML, Doll J. Tratado de geriatria e gerontologia. 3‥ ed. Ed. Guanabara Koogan, 2011.

8. Bertolucci PHF, Brucki SMD, Campacci SR, Juliano Y. O Mini Exame do Estado Mental em uma população geral: impacto da escolaridade. Arq Neuropsiquiatria, 1994, 52(1):1-7.

9. Almeida OP. Mini mental state examination and the diagnosis of dementia in Brazil. Arq Neuropsiquiatr, 1998; 56(3B): 605-12.

10. Pilger $\mathrm{C}$, Menon $\mathrm{MH}$, Mathias TAF. Características sociodemográficas e de saúde de idosos: contribuições para os serviços de saúde. Rev. Latino-Am. Enfermagem, 2011 [citado 2013 Dez 21]; 19(5): [09 telas]. Disponível em: http://www.scielo.br/pdf/rlae/v19n5/pt 22.pdf

11. Nunes MCR, Ribeiro RCL, Rosado LEFPL, Franceschini SC. Influência das características sociodemográficas e epidemiológicas na capacidade funcional de idosos residentes em Ubá, Minas Gerais. Rev Bras Fisioter. 2009; 13(5): 376-82.

12. Santos MIPO, Griep RH. Capacidade funcional de idosos atendidos em um programa do SUS em Belém (PA). Ciênc. Saúde Coletiva. 2013; 18(3):753-61.

13. Fiedler MRM, Peres KG. Capacidade funcional e fatores associados em idosos do Sul do Brasil: um estudo de base populacional. Cad. Saúde Pública. 2008; 24(2):409-15.

14. Maciel ACC, Guerra RO. Influência dos fatores biopsicossociais sobre a capacidade funcional de idosos residentes no nordeste do Brasil. Rev. bras. epidemiol. 2007; 10(2): 178-89.

15. Nunes DP, Nakatani AYK, Silveira EA, Bachion MM, Souza MR. Capacidade funcional, condições socioeconômicas e de saúde de idosos atendidos por equipes de Saúde da Família de Goiânia (GO, Brasil). Ciênc. Saúde Coletiva. 2010; 15(6): 288798.

16. Tavares DMS, Dias FA. Capacidade funcional, morbidades e qualidade de vida de idosos, Texto contexto - enferm. 2012; 21 (1):112-20.

17. Talmelli LFS, Gratão ACM, Kusumota L, Rodrigues RAP. Nível de independência funcional e déficit cognitivo em idosos com doença de Alzheimer. Rev. Esc. Enferm. USP 2010; 44 (4): 933-39.

18. Miranda LL, Pires VATN. Atuação dos enfermeiros da estratégia saúde da família na avaliação da capacidade funcional do idoso. Revista Enfermagem Integrada. 2011; 4(2): 806-20.

Recibido: 7 de abril de 2014; Aceptado: 11 de octubre de 2014

ISSN 1695-6141

๑) COPYRIGHT Servicio de Publicaciones - Universidad de Murcia 